\title{
Hipoglucemia grave recurrente secundaria a trastorno facticio por poderes
}

\section{Recurrent severe hypoglycemia secondary to factitious disorder by proxy}

\author{
Lucía Carolina Osorio-Acuña,* Mario Angulo-Mosquera,* Aura María Salazar-Solarte, ${ }^{\ddagger}$ \\ Carolina Aristizabal-Henao, ${ }^{\ddagger}$ Laura Torres-Canchala, ${ }^{\S}, *$ Juan Fernando Gómez-Castro* \\ * Fundación Valle del Lili; ${ }^{\ddagger}$ Residente de Pediatría. Facultad de Ciencias de la Salud, Universidad \\ ICESI; § Centro de Investigaciones, Fundación Valle del Lili, Cali, Colombia.
}

\begin{abstract}
RESUMEN
Objetivo: Describir un paciente pediátrico con trastorno facticio por poderes o síndrome de Münchausen. Descripción del caso: Paciente femenino de dos años con antecedente de una hermana que falleció, pero que también tuvo cuadros de hipoglucemia. Antes del ingreso más reciente, ya había registro de hospitalizaciones previas por hipoglucemia. En esta última ocasión acudió con astenia y un síncope, documentándose glucemia de $22 \mathrm{mg} / \mathrm{dL}$. Se realizaron estudios para determinar causa de hipoglucemia, pero todos fueron normales. Mediante intervención por Psiquiatría, la madre confesó administrar insulina a la paciente de manera exógena. Conclusión: El trastorno facticio por poderes es una forma grave de maltrato infantil, en donde el cuidador realiza acciones para generar morbilidad. Su identificación temprana evitará su perpetuación y un posible desenlace fatal.
\end{abstract}

Palabras clave: Hipoglucemia, Münchausen, trastorno facticio por poderes, maltrato infantil.

\section{INTRODUCCIÓN}

La hipoglucemia, definida como niveles de glucosa sérica menores a $60 \mathrm{mg} / \mathrm{dL},{ }^{1}$ es uno de los trastornos metabólicos más frecuentes en pediatría, su diagnóstico y tratamiento precoz son esenciales para la prevención de secuelas neurológicas. ${ }^{2}$ Se considera como un problema clínico común en los recién nacidos, siendo

\begin{abstract}
Objective: To describe a pediatric patient with factitious disorder by proxy or Münchausen syndrome. Case description: A two-year-old female patient with a history of a sister who died, but who also had repeated hypoglycemia. Before the most recent admission, the patient had been hospitalized for hypoglycemia on several occasions. On this last occasion, she presented with asthenia and syncope, documenting a blood glucose level of $22 \mathrm{mg} / \mathrm{dL}$. All laboratory studies to determine the cause of hypoglycemia were normal. Through psychiatric intervention, the mother confessed that she administered insulin to the patient exogenously. Conclusion: Factitious disorder by proxy is a serious form of child abuse, where the caregiver performs actions to generate morbidity. Its early identification will prevent its perpetuation and a possible fatal outcome.
\end{abstract}

Keywords: Hypoglycemia, Münchausen, factitious disorder by proxy, child abuse.

menos frecuente en lactantes, y raro en escolares y adolescentes. ${ }^{3}$

Las causas de hipoglucemia son múltiples y varían según la edad. Se incluyen errores innatos del metabolismo, problema de origen endocrinológico, o bien, efectos secundarios de medicamentos. ${ }^{1,2} \mathrm{El}$ abordaje integral y manejo oportuno disminuye la morbilidad y mortalidad. ${ }^{4}$

Correspondencia: Aura María Salazar-Solarte, E-mail: auramaria1114@gmail.com

Citar como: Osorio-Acuña LC, Angulo-Mosquera M, Salazar-Solarte AM, Aristizabal-Henao C, Torres-Canchala L, Gómez-Castro JF. Hipoglucemia grave recurrente secundaria a trastorno facticio por poderes. Rev Mex Pediatr. 2021; 88(4): 152-155. https://dx.doi.org/10.35366/102780 
En la Figura 1 se describe un algoritmo como guía para identificar la etiología de hipoglucemia no asociada con diabetes. Después de realizar estudios y no llegar a una conclusión, se deben considerar otras causas más raras, como la aplicación exógena de insulina, lo cual puede ocurrir en el trastorno facticio por poderes, también conocido como síndrome de Münchausen. ${ }^{5}$ Este trastorno representa una forma grave de maltrato infantil, en el cual el cuidador primario (que pueden o no ser los padres) hace acciones sobre los niños que le provocan síntomas que simulan una enfermedad, lo cual puede llevar a un desenlace fatal. Por fortuna, los estudios publicados son escasos, y se ha señalado que estos cuidadores sufren trastornos de personalidad, trastornos somatomorfos o depresión. ${ }^{5}$

Se ha estimado que la prevalencia del síndrome de Münchausen es aproximadamente de $0.53 \%$ en el ámbito mundial; ${ }^{6,7}$ sin embargo, dada la complejidad para llegar al diagnóstico, es probable que esta cifra esté subestimada, por lo que el conocimiento de estos casos puede alertar a los integrantes del equipo de salud para identificar de manera temprana a pacientes víctimas de este tipo de maltrato. ${ }^{8}$

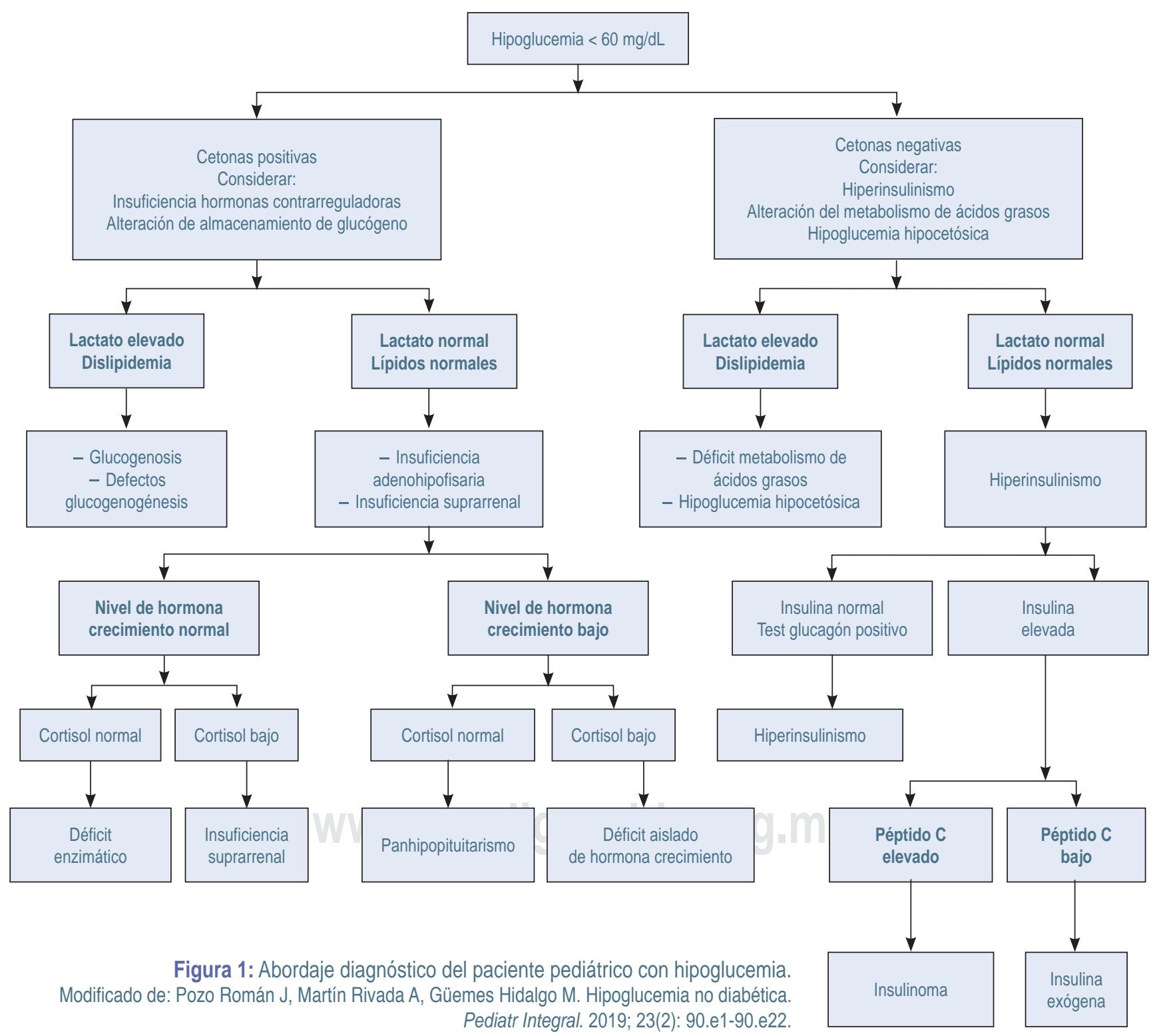


A continuación, presentamos el caso de un paciente preescolar femenino, con múltiples ingresos hospitalarios por hipoglucemia, a quien tras investigación clínica exhaustiva se logró comprobar que se trataba de un trastorno facticio por poderes en la madre.

\section{PRESENTACIÓN DEL CASO}

Femenino de dos años, producto de tercer embarazo, hija de madre diabética tipo 2. Como antecedentes de importancia, su hermana mayor fue diagnosticada con hipoglucemia no cetósica, por probable error innato del metabolismo, pero murió en su domicilio a los dos años en condiciones poco claras. Su hermano de siete años con diabetes mellitus tipo I y en tratamiento a base de insulina.

Antes del ingreso, la paciente tenía historia de varios episodios de hipoglucemia transitoria sintomática desde su nacimiento, que requirieron hospitalización donde se confirmaba el diagnóstico. Por este antecedente se encontraba en seguimiento por endocrinología, neurología y genética.

En el ingreso más reciente, acudió al servicio de Urgencias por cuadro clínico de una semana de evolución de somnolencia marcada, astenia y un síncope. La glucemia de ingreso fue de $22 \mathrm{mg} / \mathrm{dL}$. Se inició manejo con flujo metabólico alto y glucagón. En la exploración física no se encontró algún dato anormal.

Para establecer la etiología de la hipoglucemia, se solicitó toma de muestra crítica durante un episodio de hipoglucemia. Sin embargo, posterior a ocho horas de ayuno, el nivel de glucosa sérica permanecía mayor a $60 \mathrm{mg} / \mathrm{dL}$. Ante el resultado, se decidió evaluar aspectos hormonales: los niveles de insulina sérica fueron normales $(2.9 \mathrm{mU} / \mathrm{mL})$, los niveles de cortisol basal tenían buena respuesta, había ausencia de cuerpos cetónicos en sangre, y los niveles de péptido $\mathrm{C}$ estaban disminuidos $(0.3 \mathrm{ng} / \mathrm{mL})$. Con estos hallazgos se descartó un desorden hormonal, y se sospechó la presencia de un posible error innato del metabolismo.

Por Neurología Pediátrica se determinó que el neurodesarrollo era normal, lo cual, sumado a la aparición tardía de hipoglucemias y su ausencia tras el reto de hipoglucemia, permitió descartar error innato del metabolismo, por lo que se sospechó origen facticio junto al antecedente familiar de hipoglucemia

Psiquiatría Infantil solicitó entrevista con los cuidadores. La madre se negó inicialmente, pero se evidenciaron incongruencias en la información suministrada. Se entrevistó a la madre en tres ocasiones, la última en presencia de la abuela materna.
Después de confrontar a la madre, ésta manifiesta que en múltiples ocasiones había administrado insulina de acción rápida a su hijo mayor, sin indicación médica, pero con la intención de conseguir atención por parte de sus familiares. Además, confesó hacer lo mismo con la paciente. Se diagnosticó hipoglucemia por administración de insulina exógena secundario a trastorno facticio por poderes de la madre.

Se hizo notificación a trabajo social, y a entes gubernamentales por maltrato infantil. Se dio la custodia temporal a la abuela materna para el cuidado de la paciente y, subsecuentemente, ya no presentó nuevos episodios de hipoglucemia, lo que confirmó el diagnóstico.

En cuanto a la madre, se documentó cuadro depresivo distímico, rasgos prominentes de personalidad limítrofe, así como historia de disfunción familiar.

\section{DISCUSIÓN}

El síndrome de Münchausen o trastorno facticio por poderes es una condición diagnosticada cuando un cuidador, de modo intencional, fabrica o inflige una enfermedad a otro o a sí mismo para su propio beneficio. ${ }^{9}$ Es caracterizado por un patrón conductual que va desde el reporte de síntomas falsos por parte de los cuidadores (usualmente la madre) hasta la fabricación de síntomas y perpetración de daño físico (uso de venenos, ahogamiento, infusión de materia fecal, aplicación de medicamentos, entre otros) con el objetivo de asumir el rol de enfermo. ${ }^{3}$ Si bien, no hay un cuadro clínico característico, existen patrones de conducta, dentro de los que se incluyen rehospitalizaciones sin causa determinada, antecedentes similares en otros familiares, la incongruencia del relato de los cuidadores durante la anamnesis, así como la mejora espontánea del paciente.

Los médicos, y en este caso los pediatras, son a menudo los primeros profesionales que se enfrentan a la sospecha de una persona en peligro. De este modo, juegan un papel clave en la evaluación temprana y la aclaración de la sospecha diagnóstica, con una búsqueda cuidadosa de signos de alarma. ${ }^{10}$ Durante el abordaje de la paciente presentada en este reporte, se encontraron las características clásicas que se describen en este síndrome: la aparición de los signos y síntomas que sólo ocurren en presencia del cuidador, además de hermanos que compartían los mismos síntomas.

Dentro de las teorías que tratan de explicar la motivación de estas personas, están el deseo de llamar la atención, ganar gratificación emocional y reconocimiento al asumir el rol de madre dedicada, (mientras 
que, en privado, el niño(a) es sujeto de abuso físico y emocional), el cual es satisfecho al recibir la atención del médico. ${ }^{11}$ También se habla de un vínculo patológico madre-niño, asumiendo el rol de enfermo a través de su supuesto niño enfermo como una forma de pedir ayuda para sus propios problemas. ${ }^{12}$

En muchas de estas madres hay una historia de traumas y abusos en su infancia, por lo que se ha sugerido que estas personas canalizan sus inseguridades y síntomas afectivos a través de conductas abusivas hacia sus hijos. Se han reportado trastornos somatomorfos en las madres de los pacientes víctima, ${ }^{13}$ lo cual también se evidenció en la madre de esta paciente.

Es importante aclarar que el diagnóstico de este síndrome es de exclusión, después de realizar un abordaje clínico y paraclínico exhaustivo. Se debe evaluar al cuidador a través del interrogatorio, identificando inconsistencias en la narración de los síntomas, o bien, entrevistando a otros miembros de la familia que tengan cercanía con el afectado. ${ }^{14}$

Los niños víctimas de abusos deben ser separados de manera inmediata de sus cuidadores, poniéndolos a cargo del sistema de salud para que reciban manejo por parte de un grupo especializado que garantice su cuidado y bienestar, así como hacer una intervención al cuidador para el manejo de las comorbilidades mentales. ${ }^{7,15}$

\section{REFERENCIAS}

1. Gruppuso PA, Schwartz R. Hypoglycemia in children. Pediatr Rev. 1989; 11(4): 117-124. doi: 10.1542/pir.11-4-117.

2. Hoe FM. Hypoglycemia in infants and children. Adv Pediatr. 2008; 55: 367-384. doi: 10.1016/j.yapd.2008.07.008.

3. Yates G, Bass C. The perpetrators of medical child abuse (Munchausen Syndrome by Proxy) - A systematic review of 796 cases. Child Abuse Negl. 2017; 72: 45-53. doi: 10.1016/j. chiabu.2017.07.008.

4. Umpierrez G, Korytkowski M. Diabetic emergencies - ketoacidosis, hyperglycaemic hyperosmolar state and hypoglycaemia. Nat Rev Endocrinol. 2016; 12(4): 222-232. doi: 10.1038/nrendo.2016.15.
5. Hall DE, Eubanks L, Meyyazhagan LS, Kenney RD, Johnson $\mathrm{SC}$. Evaluation of covert video surveillance in the diagnosis of munchausen syndrome by proxy: lessons from 41 cases. Pediatrics. 2000; 105(6): 1305-1312. doi: 10.1542/peds.105.6.1305.

6. Noeker M, Franke I. Structured interviewing of children in suspected child endangerment cases: the German version of the revised NICHD investigative interview protocol. Bundesgesundheitsblatt Gesundheitsforschung Gesundheitsschutz. 2018; 61(12): 15871602. doi: 10.1007/s00103-018-2838-4.

7. Tatu L, Aybek S, Bogousslavsky J. Munchausen syndrome and the wide spectrum of factitious disorders. Front Neurol Neurosci. 2018; 42: 81-86. doi: 10.1159/000475682.

8. Sousa Filho D, Kanomata EY, Feldman RJ, Maluf Neto A. Munchausen syndrome and Munchausen syndrome by proxy: a narrative review. Einstein (Sao Paulo). 2017; 15(4): 516-521. doi: 10.1590/S1679-45082017MD3746.

9. Swonke ML, Smith SA, Ohlstein JF, Siddiqui F, Szeremeta W, Pine HS. Unexplained destructive nasal lesions in halfbrothers: a possible case of Munchausen syndrome by proxy. Int J Pediatr Otorhinolaryngol. 2019; 123: 75-78. doi: 10.1016/j. ijporl.2019.04.029.

10. Nathanson M. Münchausen syndrome by proxy. Child Abus Diagnostic Forensic Considerations. 2018; (26): 303-312. doi: 10.1007/978-3-319-65882-7_16.

11. Culotta PA, Isaac R, Sarpong K, Chandy B, Cruz A, DonarumaKwoh M. Nasal erosion as an uncommon sign of child abuse. Int J Pediatr Otorhinolaryngol. 2018; 108: 95-99. doi: 10.1016/j. ijporl.2018.02.024.

12. Schreier HA, Libow JA. Munchausen by proxy syndrome: a modern pediatric challenge. J Pediatr. 1994; 125(6 Pt 2): S110-S115. doi: 10.1016/s0022-3476(05)82934-8.

13. Grunwaldt LJ, Adetayo OA, Maclsaac ZM, Losee JE, Kumar AR. Successful reconstruction of complex pediatric nasal lesions: improving outcomes using dermal regenerative templates. Plast Reconstr Surg Glob Open. 2014; 2(2): e107. doi: 10.1097/ GOX.0000000000000033.

14. Tozzo P, Picozzi M, Caenazzo L. Munchausen syndrome by proxy: balancing ethical and clinical challenges for healthcare professionals ethical consideration in factitious disorders. Clin Ter. 2018; 169(3): e129-e134. doi: 10.7417/T.2018.2067.

15. Kelly M, Bursch B. Factitious disorder imposed on another. In: Driver D, Thomas S. Complex Disord Pediatr Psychiatry. A Clinician's Guide. Elsevier Inc. 2018. pp. 105-115. https://doi. org/10.1016/B978-0-323-51147-6.00009-0.

Conflicto de intereses: los autores declaran que no tienen. 